\title{
Eine neue Welle der Demokratisierung? Warum arabische Revolutionen gelingen und wann sie scheitern. Eine Zwischenbilanz
}

\author{
Kai Hafez*
}

W ährend Samuel Huntington weltweit durch seine These vom „Kampf der Kulturen“1 populär wurde und dafür sehr stark kritisiert worden ist, ist er in der Wissenschaft durch ganz andere Arbeiten bekannt geworden, unter anderem durch sein Konzept der „Wellen der Demokratisierung“, mit dem er die Durchsetzung der Demokratie im Weltmaßstab beschrieben hat. ${ }^{2}$ Nach Huntington hat sich die Demokratie in den letzten 200 Jahren wellenförmig ausgebreitet, zunächst, in einer ersten Welle in Europa und Nordamerika im 19. Jahrhundert, dann in einer zweiten Welle, die nach dem Zweiten Weltkrieg begann und bis in die 1960er Jahre hinein andauerte, und schließlich in einer dritten, noch immer anhaltenden Welle, die Mitte der 1970er Jahre in Südeuropa und Lateinamerika ihren Ausgang nahm, sich nach Ostasien ausbreitete und nach dem Ende des Ost-West-Konflikts auch Osteuropa erfasste. Zu Recht machte Huntington geltend, dass in der Geschichte selten nur ein einzelnes Land von Demokratisierungsdynamiken erfasst wurde, sondern diese häufig auf andere Länder im geografischen Umfeld übersprangen und so ganze Regionen ergriffen.

Eine solche wellenartige Ausbreitung von revolutionären Demokratisierungsprozessen sehen wir nun auch in der arabischen Welt. Sie begann in Tunesien, sprang aber recht bald schon auf Länder wie Jemen, Bahrain, Ägypten, Libyen und Syrien über. Auch in Jordanien und Marokko hat es bereits Demonstrationen gegeben. Noch ist unklar, ob wir es wirklich mit einer andauernden „Welle“ zu tun haben: Tunesien und Ägypten sind bislang die einzigen Staaten, die freie Wahlen durchgeführt haben. Allein Tunesien kann derzeit formal bereits als Demokratie angesehen werden, wohingegen in Ägypten auch nach der Parlamentswahl noch immer das Militär herrscht. Ein Präsident ist noch nicht gewählt worden. Alle anderen Länder befinden sich noch in der Transition, d.h. der Regimesturz ist noch nicht gelungen oder, wie in Libyen, freie Wahlen haben noch nicht stattgefunden. In jedem Fall aber ist in diesen Revolutionen das wellenartige Ausbreitungselement - im angelsächsischen Sprachraum auch als „Ansteckungseffekt“ (contagion) bezeichnet - allzu deutlich, sodass man vielleicht doch von einer arabischen Welle der Demokratisierung sprechen kann. All diese Versuche der begrifflichen Beschreibung greifen jedoch nur auf Metaphern zurück, die uns helfen sollen, komplexe Vorgänge zu verstehen und die natürlich nie vollständig stimmig sind: Während „Wellen“ unaufhaltsam erscheinen,

* Prof. Dr. Kai Hafez lehrt Kommunikationswissenschaft an der Universität Erfurt.

1 Huntington, Samuel P. (1993), The Clash of Civilizations?, in: Foreign Affairs 72/3, S. 22-49; ders. (1996), The Clash of Civilizations and the Remaking of World Order, New York: Simon \& Schuster.

2 Huntington, Samuel P. (1991), The Third Wave: Democratization in the Late Twentieth Century, Norman: University of Oklahoma Press. können Demokratisierungsprozesse durchaus zum Stillstand kommen oder sogar wieder rückgängig gemacht werden.

Ungeachtet aller Versuche einer begrifflichen Einordnung, ist für die politische Entwicklung in vielen arabischen Ländern im letzten Jahr typisch, dass Menschen für Regimesturz, Partizipation und Demokratisierung auf die Straße gehen. Die neuen sozialen und politischen Bewegungen sind generationen- und schichtenübergreifend. Die Dynamik verläuft in jedem Land etwas anders: Die Revolutionen in Tunesien und Ägypten verliefen weitgehend unblutig und mit den Mitteln des gewaltfreien Widerstands, so zum Teil auch im Jemen, während in Libyen, Bahrain und Syrien Gewalt vorherrschte oder vorherrscht. Warum es zu diesem Unterschieden kommt, lässt sich durchaus erklären, und der nachstehende Beitrag unternimmt den Versuch einer Zwischenbilanz der arabischen Revolutionen, deren Verläufe und Konstellationen vergleichend betrachtet werden sollen. Zwei kurze theoretische Vorbemerkungen sind dabei erforderlich. Erstens: In der politikwissenschaftlichen Demokratisierungsforschung werden Transformationen in der Regel in drei Grundphasen gegliedert: Autoritäre Phase, Transitionsphase und Konsolidierungsphase. Da sich über die Konsolidierung einer durch Wahl, Versammlungs- und Meinungsfreiheit gekennzeichneten Demokratie in der arabischen Welt derzeit noch wenig sagen lässt, beschränkt sich der Text auf die ersten beiden Phasen. Untersucht werden die Kräftekonstellationen vor den revolutionären Umbrüchen. Die Transitionsphase wird wiederum zweifach gegliedert, nämlich in die Zeit bis zum Regimesturz und in eine Periode zwischen Regimesturz und der Abhaltung erster Wahlen. Zweitens: Jede sinnvolle Reflektion über politische Umbrüche muss die klassischen Bereiche der Politik- und Sozialtheorie im Blick haben: Politische Systeme (systemtheoretischer Ansatz), sozioökonomische Basis (politische Ökonomie), Werte und politische Kultur (normative Demokratietheorie) und Akteure, neben Parteien auch soziale Bewegungen und Medien (Handlungs- und Öffentlichkeitstheorie). Transformationstheorie sollte all diese Sektoren miteinander verbinden und nicht einzelne Bereiche der Opposition oder des Staates isolieren. ${ }^{3}$

\section{Vor der Revolution - Autoritärer Staat, autoritäre Gesellschaften}

Vor den Ereignissen des sogenannten Arabischen Frühlings“ gab es drei politische Systemtypen in der arabischen bzw. isla-

3 Vgl. ähnlich: Harders, Cilja (2011), Die Umbrüche in der arabischen Welt: Zwischen Revolution und Restauration, in: Proteste, Revolutionen, Transformationen - die arabische Welt im Umbruch, hrsg. von der Arbeitsstelle Politik des Vorderen Orients, Berlin: Freie Universität, S. 10-37. 
mischen Welt: Demokratien wie etwa in Indonesien, der Türkei oder im Libanon; harte Autokratien in Ländern wie Syrien, Tunesien, Libyen oder Saudi-Arabien; und weiche Autokratien, etwa in Ägypten, Jordanien oder Marokko. Unter weichen Autokratien versteht man Herrschaftsformen, die zwar grundsätzlich diktatorisch geprägt sind, aber gewisse liberale Tendenzen aufweisen. Länder wie Ägypten und Jordanien unterschieden sich von Ländern wie Syrien und Libyen eben dadurch, dass sie zwar von einem autoritären Zentrum beherrscht wurden, dass aber dennoch zwei große Bewegungen in den letzten zwei Jahrzehnten erkennbar wurden: die Herausbildung gewisser Oppositionsparteien und -kräfte, denen ein beschränkter Freiraum gewährt wurde, und die Entstehung einer Medienöffentlichkeit, die durch relativ liberale Medienpolitiken ermöglicht wurde. Mit dem berühmten arabischen Fernsehsender Al-Jazee$r a$ verbindet sich seit anderthalb Jahrzehnten eine mehr und mehr pluralistische arabische Öffentlichkeit, in deren Zuge Hunderte von Fernsehsendern entstanden sind und sich gerade die arabische Jugend im Internet neue kommunikative Räume erschließen konnte. ${ }^{4}$ Technologische Veränderungen erzeugten einen solchen Druck auf die arabischen Regime, dass manche sich von vornherein zu einer relativ liberalen Strategie entschieden, da sie dem Wildwuchs an Satellitenschüsseln und Internetanschlüssen ohnehin nicht mehr Herr werden konnten. Die weiche Autokratie zeigte also eine schleichende Erosion staatlicher Kontrolle und eine stufenweise Stärkung der Zivilgesellschaft.

Der autoritäre arabische Staat war - und ist es in vielen Ländern noch heute - eigentlich in vielen Bereichen ein schwacher Staat, was sich auch an der sozioökonomischen Entwicklung demonstrieren lässt. Das äußere Erscheinungsbild der Stärke des arabischen Staates basiert auf der Loyalität des Militärs, auf Einheitsparteien und Klientelsystemen. Mit der rasanten Bevölkerungsexplosion in vielen arabischen Ländern aber wurde der Anteil der Profiteure an diesem System immer geringer. Der autoritäre Staat hat sich nicht nur immer mehr aus der Öffentlichkeit zurückziehen müssen, er war auch als sozialer Akteur gerade in den neuen urbanen Megastädten wie Kairo immer weniger präsent. Ägyptische Slums beispielsweise entwickelten sich in rasantem Tempo, das Regime Mubarak war kaum noch in der Lage, selbst grundlegende Infrastrukturen in Schuss zu halten, weite Teile des sozialen Lebens basierten auf der Selbstorganisation der Bürger in Netzwerken. Dennoch waren auch vor dem Sturz einige Tendenzen, vor allen Dingen in der Mittelschicht, zu beobachten, die auf positive und gegenteilige Entwicklungsdynamiken schließen ließen. Denn neben sozialer Verelendung und Proletarisierung gab und gibt es in Städten wie Kairo auch gigantische Einkaufszentren und Bauprojekte am Rande der Stadt.

Insgesamt war und ist die soziale Lage in allen autoritären arabischen Staaten - mit der Ausnahme der Erdölmonarchien schichtenübergreifend sehr explosiv. Der Sozialkontrakt, den es in der Nachkriegszeit in einigen Ländern etwa unter dem Stichwort des arabischen Sozialismus zu geben schien, ist zunehmend obsolet geworden. Die alte politologische Formel jedoch,

4 Hafez, Kai (2004), Arabisches Satellitenfernsehen - Demokratisierung ohne politische Parteien?, in: Aus Politik und Zeitgeschichte B 48, S. 17-23. wonach Demokratie erst auf hohem sozioökonomischem Niveau erfolgreich installiert werden kann, ${ }^{5}$ ist längst veraltet, wie etwa das bekannte Gegenbeispiel Indien eindrücklich vorführt. Worauf es offensichtlich ankommt, ist nicht ein bestimmtes Wohlstandsniveau, sondern ein gesteigerter Grad an sozialen Spannungen, Disparitäten und Verschiebungen.

Aber die ökonomische Deutung der derzeitigen arabischen Revolution ist unzureichend, denn ökonomischer Druck kann, wie die Geschichte gezeigt hat, auch zu Autokratisierung, ja gar zu Faschismus, führen. Wieso aber hat die arabische Welt trotz ökonomischer Dauerkrise eine Demokratiebewegung hervorgebracht?

Zur Beantwortung dieser Frage muss man sich der Entwicklung der politischen Kultur in dieser Region zuwenden. Man vergisst gerne, dass arabische Staaten und Iran im 20. Jahrhundert gewisse, wenn auch kurzlebige, Erfahrungen mit der Demokratie gemacht haben. In Iran fand 1907 eine konstitutionelle Revolution statt, und Ägypten war in der Zwischenkriegszeit und kurz nach dem Zweiten Weltkrieg (1919-1952) eine Wahldemokratie. In ihr hatten zwar der König wie auch die ehemalige britische Kolonialmacht einen erheblichen Einfluss, aber dennoch kann gerade Ägypten auf eine gewisse Demokratieerfahrung zurückblicken. Die Wafd-Partei, die bis heute existiert, besteht seit der Zeit dieser frühen ägyptischen Experimente. Das nationale Frauenwahlrecht wurde in Ägypten 1956 eingeführt, nur wenige Jahre nach Ländern wie Frankreich (1945) oder Italien (1946). Zahlreiche politische Meinungsumfragen und empirische Untersuchungen zur politischen Kultur der letzten zehn Jahre haben gezeigt, dass die Mehrheit in den arabischen Ländern Demokratie befürwortet. ${ }^{6}$ Man brauchte also angesichts der jüngeren demokratischen Umbrüche in der arabischen Welt überhaupt nicht überrascht zu sein. Wir wissen seit Jahrzehnten, dass die arabischen Bevölkerungen zwei Dinge wollen: Demokratie und Religion - auf den letzten Aspekt komme ich später noch zurück.

Die arabische politische Kultur befand und befindet sich insgesamt in einer ganz außergewöhnlichen Position der Transformation, die aber im Westen oft übersehen worden ist, weil sie nicht zu den üblichen Stereotypen der arabischen islamischen Welt passt. Während Transformationsforscher in der Regel davon ausgehen, dass sich eine demokratische politische Kultur erst nach einem Systemwechsel langsam durch Erziehung usw. herausbildet, war und ist dies in der arabischen Welt viel früher der Fall, nämlich bereits in der autoritären Phase. Gerade moderne grenzüberschreitende Medien des Westens, insbesondere aber panarabische Medien haben zu einer kommunikativen Wende beigetragen. Araber waren in den letzten Jahren nicht

5 Lipset, Seymour Martin (1960), Political Man, London: Heineman.

6 Esposito, John L./Dalia Mogahed (2007), Who Speaks for Islam? What a Billion Muslims really Think. Based on Gallup's World Poll - the Largest Study of its Kind, New York: Gallup; Tessler, Mark (2000), Democratic Concern and Islamic Resurgence: Converging Dimensions of the Arab World's Political Agenda, in: Howard Handelman/Mark Tessler (eds.), Democracy and its Limits. Lessons from Asia, Latin America, and the Middle East, Notre Dame: University of Notre Dame Press, p. 262-289; ders. (2002), Islam and Democracy in the Middle East. The Impact of Religious Orientations on Attitudes toward Democracy in four Arab Countries, in: Comparative Politics 2, p. 337-354; ders. (2003), The Influence of Islam on Attitudes toward Democracy in Morocco and Algeria, in: Amin Saikal/Albrecht Schnabel (eds.), Democratization in the Middle East: Experiences, Struggles, Challenges, Tokyo, etc: United Nations University Press, p. 103-123. 
isoliert und abgeschirmt, wie dies überwiegend im früheren Ostblock der Fall war, sondern sie waren gut informiert und konnten ihre politischen Werte auch in einer Zeit entwickeln, als der autoritäre arabische Staat noch fest im Sattel saß. Ähnliche Entwicklungen kennen wir auch aus der europäischen Geschichte, etwa im Zeitalter der europäischen Reformationen, die ohne die Erfindung des Buchdrucks durch Johannes Gutenberg und die Verbreitung protestantischer Schriften nicht möglich gewesen wären. Man konnte in den letzten Jahrzehnten in den Medien und im Internet die Aufbruchsstimmung, die Kraft und das neue Selbstbewusstsein spüren, das sich in der arabischen Welt formierte.

Alle strukturellen Voraussetzungen eines erodierenden autoritären Staates, hoher sozialer Druck und demokratiefreundliche politische Kulturen reichen jedoch nicht aus, wenn es keine Akteure und Kräfte in einer Gesellschaft gibt, die diese Druckfaktoren in produktive politische Handlungen umsetzen. Die Politikwissenschaft fragt daher mit Nachdruck nach der Konfliktfähigkeit von Oppositionskräften. Erst sie ist geeignet, strukturelle Voraussetzungen in effektive politische Transformation umzusetzen. Wie aber sah es mit der Konfliktfähigkeit in arabischen Ländern vor den politischen Umbrüchen des Arabischen Frühlings aus? Und wieso gelingt in manchen Ländern, was in anderen scheitert - der Regimesturz?

Politische Parteien existieren in den meisten Ländern, wenn überhaupt, vielfach in der Halblegalität. Nationalisten, Islamisten, Sozialisten: Die Programme der meisten politischen Parteien sind unter den Bedingungen autoritärer Herrschaft vielfach nicht klar demokratisch ausgerichtet. Manche Parteien agieren minimalistisch, quasi als „Blockparteien“, ähnlich wie in der ehemaligen DDR: Sie lassen sich für die Rituale der Pseudodemokratie - Scheinwahlen, Scheinparlamente - vereinnahmen; andere sind relativ wenig institutionalisierte Ein-Mann-Parteien. Das Ansehen politischer Parteien in der arabischen Welt war in den letzten Jahrzehnten in der Regel nicht sehr hoch, gerade junge Leute wendeten sich von ihren verkrusteten Strukturen ab und suchten nach neuen Wegen der politischen Aktivität.

Eine Ausnahmeerscheinung waren immer die Islamisten, von denen ein kleinerer Teil sich dem bewaffneten Kampf im Untergrund zuwendete, während die größeren Organisationen wie etwa die Muslimbrüder in Ägypten oder Marokkos Parti de la Justice et du Développement (PJD), die ägyptische Al-Wasat (Zentristen), Jemens Islah (Reformpartei), Jordaniens Islamische Aktionsfront, Kuwaits Islamische Konstitutionsbewegung und Bahrains Al-Wifaq (Die Übereinstimmung) sich zwar gerade in der Frage des Säkularismus eine gewisse Radikalität erhielten, eine antiautoritäre Ausstrahlung, die sie gleichwohl nicht dazu verleitete, von sich aus einen Regimeumsturz zu versuchen. Islamisten betrieben und betreiben in autoritären Staaten aktive Sozialarbeit, sie brachen in das Vakuum ein, das der schwächelnde autoritäre Sozialstaat hinterließ. Der Staat agierte häufig härter gegen die säkulare Opposition als gegen die Islamisten, denn letztere ließen sich gerade dem Ausland gegenüber als Selbstlegitimation der eigenen autoritären Herrschaft verkaufen. Die Rechnung ging aber letztendlich nicht auf: Das säkulare Vertretungsmonopol des Staates stärkte lediglich die religiöse Opposition.
Man muss diese spezifische Problematik am Rande der Weltgesellschaft verstehen: Während Säkularität etwa in Europa einen Fortschritt an Freiheit gegenüber kirchlichen und anderen repressiven Institutionen gebracht hat, wurde sie vom autoritären arabischen Staat in die Region importiert und zum Bestandteil einer repressiven Ideologie und jahrzehntelanger Unterdrückung gemacht. ${ }^{7}$ Sich gegen Säkularität zu wenden und mit den Islamisten zu sympathisieren, hatte und hat daher nicht selten einen gewissen antiautoritären Charme. Die Frage, ob Islamisten demokratieverträglich sind, lässt sich denn auch nur sehr differenziert beantworten. Eine Studie des Carnegie Endowment for Peace spricht in diesem Zusammenhang von ideologischen „Grauzonen“. ${ }^{8}$ Gewaltenteilung, politische Parteien und Parlamentarismus sind für die Menschen in den islamistischen Organisationen in der arabischen Welt jederzeit akzeptabel, sie sind in diesem Sinne antiautoritär und kanalisieren den Partizipationswillen der Menschen in der arabischen Welt, die des korrupten Missmanagements diktatorischer Regime überdrüssig sind und ihre Gesellschaften selbst gestalten wollen. Das Hauptproblem des Islamismus ist nicht die institutionelle Demokratie oder die Gefahr einer Religionsdiktatur nach dem Vorbild Irans - eines Systems der Herrschaft der Theologen, das es übrigens in der islamischen Welt so vorher noch nie gegeben hat -, sondern das Hauptproblem rankt sich um die Frage der Säkularität. Die Gleichberechtigung von Angehörigen unterschiedlicher Religionen oder die Gleichberechtigung von Mann und Frau vor dem Gesetz stehen für Islamisten im Widerspruch zur religiösen Werteordnung, und es ist insofern berechtigt, sie als „Fundamentalisten“ zu bezeichnen.

Allerdings gibt es im Lager des politischen Islam mittlerweile auch Strömungen wie die ägyptische Wasatiyya (wasat arab.: Mitte), die islamischen Zentristen, für die Säkularität kein Problem mehr darstellt und die sich zu einem Reformislam bekennen. Die „Christdemokratisierung“ des Islams ist also in Teilen schon erfolgt, in anderen Teilen steht sie noch aus und wird wohl noch auf sich warten lassen. In diesem Sinne ist es auch berechtigt von moderaten Islamisten zu sprechen, die vor den Regimeumstürzen in Tunesien und Ägypten eine wichtige Rolle spielten und dies in Ländern wie Marokko und Jordanien auch heute noch tun. Die Ablehnung des Säkularismus ist ein radikaler Kampfslogan dieser Gruppierungen, die den Rahmen einer „islamischen Demokratie“ gestalten wollen. Ob sie damit Erfolg haben werden, hängt nicht allein von ihnen ab. Denn trotz aller Erfolge des Islamismus in der Opposition waren bzw. sind Islamisten alleine nicht zum Regimesturz fähig. Was vor den aktuellen Revolutionen jahrzehntelang fehlte, war ein lagerübergreifendes Bündnis mit häufig säkularen Bevölkerungsteilen.

Welches Fazit können wir also hinsichtlich der Voraussetzungen des Systemwechsels zur Demokratie vor den Ereignissen des Arabischen Frühlings ziehen? Der autoritäre arabische Staat war oder ist in Teilen ein schwacher Staat, die arabische politische Kultur hat sich wesentlich dynamischer entwickelt als

7 Hafez, Kai (2010), Radicalism and Political Reform in the Islamic and Western Worlds, Cambridge et al.: Cambridge University Press.

8 Brown, Nathan J./Amr Hamzawy/Marina Ottaway (2006), Islamist Movements and the Democratic Process in the Arab World: Exploring the Grey Zones, Carnegie Papers Nr. 67, Washington, D.C.: Carnegie Endowment for International Peace. 
ihre politischen Systeme. Massenmedien und alle möglichen Formen neuer Medien traten als neue politische Akteure auf, sie übernahmen Ersatzfunktionen für die politischen Parteien, die - und dies war das größte Manko bisher - trotz aller vorhandener Ansätze zur Opposition nur bedingt bündnis- und konfliktfähig waren.

\section{Transition, erste Phase: bis zum Regimesturz}

Mit den Ereignissen des „Arabischen Frühlings“ tritt nun ein neuer Akteur auf die arabische Bühne: die sozialen und politischen Bewegungen. Ihr Genie ist es, dass sie die ideologischen Gräben der Vergangenheit, die vor allen Dingen zwischen Säkularisten und Islamisten bestanden, zu überbrücken vermögen. Für die Aufstände des „Arabischen Frühlings“, die sich von Tunesien aus über weite Teile der arabischen Welt ausgebreitet haben, ist die Abwesenheit von parteipolitischen Slogans typisch. Menschen demonstrieren nicht für eine bestimmte politische Richtung, sondern sie sind vereint gegen die jeweiligen Diktatoren. Solche Bündnisse gab es zuvor in der arabischen Welt nur sehr selten, man denke etwa an die Kifaya-Bewegung, die Mitte des letzten Jahrzehnts in Ägypten kleinere Demonstrationen zustande brachte und dabei säkulare und islamistische Kräfte vereinte. Die Bündnispolitik aber war insgesamt schwach ausgeprägt und es ist den arabischen Diktatoren immer wieder gelungen, die unterschiedlichen Oppositionskräfte gegeneinander auszuspielen. Damit ist seit etwa einem Jahr Schluss. Politische Bewegungen haben sich über die Grenzen politischer Lager hinweg gebildet und sie vereinen dabei unterschiedliche Schichten und Generationen. Trotz der im Durchschnitt sehr jungen arabischen Bevölkerung wäre es sicherlich verkürzt, die arabischen Umbrüche als die Revolution der „Baby-Boomer“ bezeichnen zu wollen. ${ }^{9}$ Junge Menschen stehen zwar, wie bei allen welthistorischen Revolutionen, häufig in der ersten Reihe, aber Menschen aller Generationen sind gegen die autoritären Regime in der Region vereint. Ohne die Teilnahme eines großen Teils des ägyptischen Bürgertums etwa wäre der Sturz des Mubarak-Regimes sicherlich niemals gelungen. Vielleicht erstmals in der jüngeren Geschichte Ägyptens war man geradezu glücklich über das enorme Bevölkerungswachstum dieses Landes, das normalerweise als Hauptursache der sozialen Verelendung gilt. In der akuten Umbruchsituation aber erdrückte die zahlenmäßig starke Bevölkerung das autoritäre Regime förmlich.

Das Genie der tunesischen und ägyptischen Revolutionen, aber auch des libyschen Widerstandskampfes, wenngleich mit weniger Erfolg (s.u.), bestand nicht nur in der spontanen Bündnisbildung, sondern auch in der Ad-hoc-Organisationsfähigkeit. Bestechend war der nie geübte, aber irgendwie gekonnte gewaltfreie Widerstand. Tunesien und Ägypten waren trotz mancher Opfer im historischen Maßstab unblutige Revolutionen, geprägt von diszipliniertem Massenprotest, man denke nur an die großen Gruppen betender Demonstranten auf dem TahrirPlatz, inmitten von Panzern, Sit-ins und Blockaden. Religion waren nicht dominant, wo sie aber in Erscheinung trat, war sie

9 Perthes, Volker, Arabischer Frühling: Aufstand der Baby-Boomer, Der Tagesspiegel, 28. August 2011. nicht fundamentalistisch-aggressiv, sondern hochgradig liberal-zivilisiert.

Zu den neuen politischen Vergemeinschaftungsprozessen trugen viele sogenannte Social Movement Organizations bei. Soziale Bewegungen sind nämlich keineswegs chaotische Gebilde, sondern sie werden von spontan gebildeten Akteurseinheiten geprägt, wobei im aktuellen Fall vor allen Dingen das Internet eine erhebliche Rolle spielte. Facebook-Gruppen und andere Gemeinschaften organisierten den Protest über das Internet. Der Begriff der „Facebook-Revolution“ gehört aber ebenso wie der der "Jugendrevolution“ zu den aktuellen Mythen der arabischen Revolution und er ist selbst unter arabischen Bloggern höchst umstritten. Das Internet war bei den Umbrüchen in Tunesien und Ägypten gerade in den ersten Tagen bedeutsam, dann allerdings wurde es ebenso wie die gesamte Mobiltelefonie vom Regime abgestellt. Der Dynamik des Protests tat dies jedoch keinen Abbruch. Hinter dem Tahrir-Platz in Kairo und in zahlreichen anderen Städten Ägyptens etwa bildeten sich, wie vorher bereits in Tunesien, kleine Demonstrationsgruppen, die durch Mund-zu-Mund-Propaganda Menschen aus ihren Häusern riefen. Dabei entwickelten sie ganz traditionelle Formen der Versammlungskommunikation, wie sie Menschen schon seit Jahrtausenden praktizieren. Diese Prozesse werden von uns gerne übersehen, wir konzentrieren uns auf technische Innovationen, auf die neuen Medien, die häufig vom Westen geprägt worden sind und die uns insofern eine eigene Rolle in diesen welthistorischen Entwicklungen zu geben scheinen. Man muss jedoch daran erinnern, dass demokratische Umbrüche auch schon vor etwa zwanzig Jahren in Lateinamerika ohne das Internet möglich gewesen sind. Und auch in der arabischen Welt hat das Internet die Transition lediglich angeschoben, die Revolutionen aber haben schnell eine Eigendynamik entwickelt.

Nicht zu verkennen ist aber auch, dass ohne den Einsatz der großen Medien eine erfolgreiche Mobilisierung der Bevölkerungen Tunesiens und Ägyptens nicht möglich gewesen wäre. Nach der Abschaltung des Internets machte der Fernsehsender Al-Jazeera aus dem Sturm der Proteste einen regelrechten Tsunami, Al-Jazeera holte die Mittelschichten aus ihren Wohnungen. Insgesamt ließ sich also bei den bisherigen politischen Umbrüchen eine relativ typische Abfolge erkennen: Am Anfang waren die kleinen Revolutionsmedien, etwa übr das Internet, von großer Bedeutung, sie brachten den Stein ins Rollen, dann übernahmen die großen Massenmedien das Ruder. Nach dem erfolgten Regimesturz erlitten die transnationalen panarabischen Medien einen relativen Bedeutungsverlust und Menschen gingen wieder mehr zu ihren nationalen Medien über, bei denen nunmehr die Hoffnung bestehen konnte, dass hier noch mehr Meinungsfreiheit einzog. Insgesamt aber ist es eindeutig, dass es zum Arabischen Frühling erst dadurch kommen konnte, dass sich aus bis dahin isolierten politischen Akteuren neue soziale Bewegungen und politische Bündnisse bildeten und dass diese mit Hilfe alter Kommunikation und neuen Medien Wege fanden, die arabischen Zivilgesellschaften gegen die Diktatur zu vereinen.

Warum aber gelang in Ländern wie Tunesien und Ägypten was zumindest bisher in Staaten wie Bahrain, Syrien und Jemen nicht und in Libyen erst mit westlicher Hilfe möglich wurde? Die Antwort ist, dass die Ausgangslage in den unterschiedlichen 
Bereichen der Transformation (Staat, Ökonomie, Werte, Opposition) vielfach sehr unterschiedlich war. Es existieren ganz andere mediale Verhältnisse. Ägypten war vor Ausbruch der Revolution bereits das Zentrum der arabischen Bloggerbewegung, ganz anders als Libyen oder Syrien mit einer restriktiven Internetpolitik. Zudem fanden nicht alle arabischen Aufstände bei den panarabischen Fernsehsendern die gleiche Beachtung: Über den Aufstand von Bahrain etwa wurde von Al-Jazeera in vielen entscheidenden Momenten kaum berichtet, da bei aller Freiheit, die in diesem Sender herrscht, die Innenpolitik der Golfemirate eine Tabuzone darstellt.

In den Ländern zeigen sich auch ganz unterschiedliche soziale Bedingungen. Die sogenannte „Rentenökonomie“ der Erdölstaaten erlaubt einen hohen Wohlstand für weite Teile der Bevölkerungen, der soziale Druck auf die Einheimischen ist vergleichsweise gering, auch wenn gerade schiitische Bevölkerungsminderheiten häufig eine vernachlässigte Unterschicht darstellen. In den Rentenökonomien aber zeigt sich, dass der amerikanische Leitspruch „No taxation without representation“ auch umgekehrt gelten kann, denn in Staaten ohne substanzielle Besteuerung ist offensichtlich auch der Drang der Bevölkerungen zu mehr Teilhabe an der Macht nur sehr begrenzt. Ein anderer sozialer Unterschied zwischen den arabischen Staaten liegt im Verhältnis von Bevölkerungsgröße zu Landesterritorium. Ägypten besitzt etwa das Zehnfache der Bevölkerungszahl von Libyen, aber auf einer ähnlich großen Landesfläche, was in Libyen ungünstige Bedingungen für einen spontanen Aufstand in der Fläche erzeugt hat. Arabische Diktatoren können zudem in manchen Staaten - Bahrain, Syrien usw. - die Karte des religiös-ethnischen Konflikts ausspielen: In Bahrain dominierte in vergangenen Jahrzehnten der schiitische Bevölkerungsprotest, in Syrien kann man Kurden, Alawiten und Sunniten gegeneinander ausspielen, und in Libyen ist die Stammesloyalität noch immer stark. Ein konsolidierter Nationalstaat ist also eine günstige Bedingung für einen erfolgreichen Aufstand. Sollte eine Demokratisierung in Jemen gelingen, so wäre dies vielleicht ein erstes Beispiel dafür, dass demokratischer Wandel auch in stark stämmisch geprägten Gesellschaften möglich ist. Auch für Stämme ist die Demokratie häufig der einzige Weg eines friedlichen Ausgleichs. Außerdem sind sogenannte neopatriarchalische Gesellschaften eben nicht nur traditionell zu verstehen. Menschen pflegen in diesen Ländern nicht nur traditionelle Loyalitäten, sondern sie sind zugleich Mitglieder in modernen Institutionen und üben moderne Berufe aus, sodass ihre soziale Orientierung durchaus komplex sein kann. Der „Scheich mit dem Handy“ symbolisiert zudem sinnbildlich die Schnittstelle zwischen Tradition und Moderne.

$\mathrm{Zu}$ den Bedingungen, die in den verschiedenen Staaten Revolutionen begünstigen oder erschweren, gehört auch die sehr unterschiedliche staatliche Militärkultur. In Ägypten etwa beobachtete das Militär die Aufstandsentwicklung, es verhielt sich opportunistisch und schlug sich auf die Seite der Bevölkerung als es merkte, dass diese sich gegen das Regime Mubarak durchsetzen würde. In Ägypten galt und gilt das Militär als eine Einrichtung des Volkes, das Aufstiegsmöglichkeiten auch für untere Schichten bereit hält, anders als die ägyptische Polizei, deren Hierarchien sehr viel regimetreuer waren. In Län- dern wie Libyen, Jemen oder Syrien hingegen ist das Militär eng an das jeweilige autoritäre Regime gebunden, es wird systematisch von der Bevölkerung ferngehalten, indem bewußt Minderheiten wie die herrschenden Alawiten in Syrien oder ausländische Legionäre wie in Libyen eingeschleust werden.

Die These allerdings, dass in den arabischen Ländern auch unterschiedliche politische Kulturen existierten, wobei insbesondere die Monarchien in Marokko und Jordanien deswegen stabiler seien, weil sie in der Bevölkerung als legitimer gelten, ${ }^{10}$ ist weitgehend falsch. Zum einen haben in diesen Ländern bereits Demonstrationen stattgefunden, zum anderen will die Mehrheit sicherlich auch dort zumindest den Übergang zu einer echten konstitutionellen Demokratie schaffen, und dies wäre nach allen Maßstäben der Politikwissenschaft ein echter Regimewechsel, denn die bestehenden Monarchien können zwar in gewissem Maße als „aufgeklärt“ gelten, sie sind jedoch nicht demokratisch oder in irgendeiner Weise mit dem politischen System der konstitutionellen Monarchie, etwa nach dem Vorbild Englands, vergleichbar. Selbst wenn die Mehrzahl der Bevölkerung in Marokko und Jordanien den Wunsch hegt, ihre Könige zu behalten, so wünscht sie sich diese als repräsentierende Figuren, als Symbole des Staates und der Nation, möglicherweise sogar der Religion, nicht jedoch als Schutzpatrone korrupter Regierungen.

\section{Transition Phase zwei: bis zu den Wahlen}

Wo stehen Tunesien und Ägypten ein knappes Jahr nach dem Sturz ihrer Diktatur? Es ist schwer, hier die Übersicht zu behalten, da die Ereignisse sich vielfach geradezu überschlagen. Als Leitmotiv für eine Zwischenbilanz aber soll folgender Kerngedanke dienen: Das Gelingen des Regimesturzes in Tunesien und Ägypten basierte auf dem Zusammenspiel von gesellschaftlichen Werten und Einstellungen mit oppositionellen Akteuren und Bewegungen und mit den Medien als kommunikativem Band. Nur so konnte ein ausreichender gesellschaftlicher Druck auf die bereits schwächelnden politischen Systeme ausgeübt werden. Wo im Prozess der Transition eines dieser Elemente verloren geht, kann der Staat eine „zweite Chance“ und eine Gelegenheit zur Konterrevolution wittern.

Kann also der hohe Transformationsdruck in Ägypten und Tunesien aufrechterhalten werden? Als eine Art vorweggenommenes Fazit lässt sich sagen, dass sich bei vielen Beobachtern im Westen nach der Euphorie des Winters und des Frühjahrs bereits wieder zu großer Pessimismus breitzumachen scheint. Viele Entwicklungen in Tunesien und Ägypten im Jahr 2011 waren aber nicht unerwartet, der Übergang zur Demokratie ist ebenso holprig, wie dies in anderen Regionen der Welt, insbesondere in Lateinamerika, der Fall gewesen ist. Dennoch befinden wir uns noch immer im Zyklus einer zwar nicht idealen, aber weitgehend normalen Transformation, die nachfolgend erneut mit dem Blick auf die unterschiedlichen Sektoren des Wandels aufgearbeitet werden soll.

10 Asseburg, Muriel (2011), Der arabische Frühling. Herausforderung und Chance für die deutsche und europäische Politik, Berlin: Stiftung Wissenschaft und Politik, S. 14 f. 
Was die Situation des Staates angeht, so hat Tunesien mit den Parlamentswahlen bereits den Übergang zur Demokratie geschafft. Die Situation in Ägypten ist etwas schwieriger, da das Militär immer noch herrscht, obwohl auch hier bereits Parlamentswahlen stattgefunden haben. Erst durch vehementen Druck der „ägyptischen Straße“ ist es im Herbst 2011 gelungen, den Militärs zumindest das Versprechen abzuringen, die Präsidentschaftswahlen früher als geplant Mitte 2012 durchzuführen, was demnach auch das Datum der endgültigen Machtübergabe sein müsste. Die bisherige Performance des Militärs war uneinheitlich. Auf der einen Seite wurde der frühere Präsident Mubarak verhaftet und vor Gericht gestellt, und zahlreiche richtige Maßnahmen wurden initiiert. So befindet sich fast kein Vertreter des alten Regimes mehr im Hohen Militärrat, und die juristische Aufarbeitung der Ära Mubarak hat begonnen, was verhältnismäßig zügig ist, wenn man etwa an das Vorbild Chiles denkt, das sich hierfür Jahrzehnte Zeit ließ. Die ägyptische Regierungspartei wurde aufgelöst und viele korrupte Eliten haben bereits das Land verlassen. Auf der anderen Seite aber zeigen sich auch Züge einer möglichen Konterrevolution: Revolutionäre wurden verhaftet und durch Militärtribunale abgeurteilt, und Amnesty International klagt die Interimsregierung an, systematisch zu foltern. ${ }^{11}$ Die Rolle des Militärs beim Übergriff auf die israelische Botschaft in Kairo und bei Übergriffen auf Kopten ist äußerst zwielichtig. Das Militär ordnete die Stürmung des Büros des Fernsehsender Al-Jazeera in Kairo an. Die Spannungen zwischen den sozialen und politischen Bewegungen und dem Militär wachsen. Möglicherweise lässt sich hier sogar eine gewisse Paradoxie erkennen, wonach gerade die frühe juristische Aufarbeitung der Diktatur die Angst bei den alten Eliten in Ägypten gesteigert hat und auch den Hang des ägyptischen Militärs, die Demokratie auszubremsen. Vielleicht wäre es sinnvoll, Signale einer möglichen Amnestie auszusenden, denn der Aufbau der neuen Demokratie ist sicherlich wichtiger als kurzfristige Abrechnungen, auch wenn dies dem moralischen Empfinden widersprechen mag.

Die Gefahr der Bildung einer Militärjunta ist dennoch in Ägypten äußerst gering; das Militär wird das Land nicht dauerhaft selbst regieren wollen, dafür sind die Probleme des Landes zu komplex. Noch ist allerdings nicht klar, welche Entwicklung das ägyptische Militär durchlaufen wird. Während das türkische Militär jahrzehntelang ein antidemokratischer Hüter der säkularen Ordnung Atatürks war, ist das ägyptische Militär sicherlich nicht so laizistisch geprägt, sodass es wahrscheinlicher ist, dass es sich zum Hüter einer demokratischen Ordnung machen wird, auch wenn diese im Falle der Übernahme der Regierungsmehrheit durch Islamisten keine „lupenreine“ säkulare Demokratie wäre. Leider ist es nicht gelungen, eine säkulare Verfassung vor den Parlamentswahlen zu verabschieden: Die Übergangsreformen der sogenannten Bishri-Kommission im Frühjahr waren nur halbherzig und fragwürdig. Das islamische Recht (Scharia) als Quelle des Rechts blieb erhalten, eine strikte Säkularität ist mittelfristig unwahrscheinlich. Bei dem Besuch des türkischen Premierministers Tayyip Erdogan im Sommer 2011 in Ägypten konnte man trotz des enormen Zuspruchs, den er erhielt, in der Öffentlichkeit auch Kritik am laizistischen Kurs der Türkei hören. Das Militär könnte also als

11 Vgl. amnesty.org/en/region/egypt.
Schutzpatron einer „islamischen Demokratie“ relativ sicher sein, den konservativen Teil der Bevölkerung auf seiner Seite zu haben.

Es gab und gibt in Tunesien und Ägypten noch immer Probleme mit der Medienfreiheit. Zwar wurden viele neue Medien zugelassen, aber gerade in Ägypten ist die Staatsnähe des Fernsehens noch sehr ausgeprägt. Allerdings gibt es auch neue unabhängige Fernsehsender wie etwa Al-Tahrir-TV, die innerhalb kürzester Zeit beliebt geworden sind. Wie sich die Verhältnisse verändert haben, zeigt paradigmatisch auch eine neue Unterhaltungsshow im ägyptischen Fernsehen, bei der sich eine Frau aus den Armenvierteln Kairos zum neuen Kochstar entwickelt hat. Diese neue Form der Unterhaltung macht soziale Fragen transparenter als dies früher möglich war, Call-in-Sequenzen, bei denen sich die Köchin mit ihren Mitbürgern unterhält und streitet und bei denen sie die Würde der Armut mit Nachdruck verteidigt, zeigen eine weitere Liberalisierung der Öffentlichkeit an. In der ägyptischen Cartoon-Kultur ist Kritik am Militärchef Hussein Tantawi sehr verbreitet - aber für die gleiche Kritik kann man eben auch vor einem Militärtribunal enden. Bei der demokratischen Transformation muss nach dem Regimesturz eine Verlagerung von kleinen in große Medien stattfinden - und diese Verlagerung ist in Ägypten teilweise blockiert. Eine neue Regierung und ein neues ägyptisches Parlament müssen den Weg zur Meinungsfreiheit konsequent frei machen, daran werden sie zu messen sein.

Wichtig ist, dass sich in Tunesien und Ägypten die Parteienlandschaft weiter ausdifferenziert und konsolidiert. Die dezentralen Strukturen der sozialen Bewegungen waren gut für die erste Transitionsphase, sie haben die Opposition unberechenbar gemacht und den Regimen die Gegenwehr erschwert; sie sind jedoch nur teilweise nützlich für die zweite Transitionsphase und für die Durchführung demokratischer Wahlen. Beharrende Gewalten eines alten Regimes haben alleine noch nie ein System reformiert, ebenso wenig tun dies soziale Bewegungen, sondern es bedarf eines funktionierenden parlamentarischen Raums. Bislang sind die Islamisten in Tunesien und in Ägypten die am besten organisierten politischen Kräfte, sie haben eine lange Organisationsgeschichte vorzuweisen. Das Problem, vor dem beide Länder in jüngster Zeit gestanden haben, ist, dass auf der einen Seite säkulare Parteien eine längere Entwicklungsperiode bräuchten, um sich auf Wahlen vorzubereiten, auf der anderen Seite Wahlen schnellstmöglich durchgeführt werden mussten, um den alten Regimen keine Chance zur Konterrevolution zu lassen. In beiden Ländern hat es zahlreiche Parteigründungen gegeben, aber die islamistischen Kräfte haben bei den Parlamentswahlen 2011 Mehrheiten erzielt.

Unter dem Strich haben bei den ersten Parlamentswahlen vor allem alte Parteien wie die Muslimbrüder in Ägypten profitiert. Dass man deshalb noch längst keine Angst vor einer islamischen Diktatur haben muss, ist oben bereits erörtert worden. Die Islamisten Tunesiens und Ägyptens bekennen sich zur Demokratie. Sie verfügen auch nicht über geistliche Führungsfiguren, wie etwa einst der iranische Ajatollah Khomeini eine war. Weder Tunesien noch Ägypten sind Erdölstaaten mit unabhängigen Ressourcen, sie bedürfen enger Beziehungen zum Westen, um durch Wirtschaftshilfe und Tourismus das Bruttosozialprodukt zu steigern und die Haushalte zu konsoli- 
dieren. Man muss an dieser Stelle einmal deutlich sagen, dass in der Formel von der „liberalen Demokratie“ zwei Bestandteile enthalten sind: die liberale Menschenrechtsidee und die demokratische Ordnung. Beide aber sind nicht identisch, denn zu den Kernbeständen einer Demokratie gehören im Grunde zunächst einmal lediglich freie Wahlen, Versammlungsfreiheit und Meinungsfreiheit. Was aber machen wir in einer Situation, in der sich eine Mehrheit einer Bevölkerung für die Ungleichbehandlung von Religionen oder für eine rechtliche Differenz zwischen Mann und Frau entscheidet? Es ist ziemlich sicher davon auszugehen, dass die großen islamistischen Parteien in der arabischen Welt heute als Demokraten angesehen werden können, auch wenn sie keine „liberalen Demokraten“ sind. Es wird an den säkularen Kräften in diesen Gesellschaften sein, sich diese Gleichheitsrechte im Rahmen der demokratischen Ordnung Stück für Stück zu erkämpfen - ganz ähnlich wie dies auch in westlichen Demokratien der Fall war, wo es bis heute in vielen Fällen keine Gleichberechtigung von religiösen Minderheiten gibt - man denke nur an die Privilegien der christlichen Kirchen in Deutschland.

Die Hauptbedrohung der Demokratie aber geht derzeit nicht von den Islamisten aus, sondern von den beharrenden Gewalten im und hinter dem Militär. Wir müssen vorsichtig sein, hier nicht der Panikmache des Militärs zu erliegen. Westliche Staaten haben jahrzehntelang autoritäre Strukturen in der arabischen Welt aus Angst vor islamischen Staaten unterstützt, oft jedoch auch aus opportunistischer Profitgier, wobei Islamisten als nützlicher Prellbock für westliche Außenpolitiker und autoritäre Staatsführer in der Region herhalten mussten. ${ }^{12}$

Für die Weiterentwicklung der arabischen Demokratien wird es von entscheidender Bedeutung sein, dass neben der pluralistischen Besetzung der Parlamente soziale Bewegungen als außerparlamentarische Opposition aktiv bleiben. Trotz aller Gewalt, die im Jahr 2011 auch in Ländern wie Tunesien und Ägypten immer wieder aufblitzte, ist die Tatsache, dass in diesen Ländern nicht die politischen Systeme die Menschen, sondern die Menschen die politischen Systeme vor sich hertreiben und sie zu verbessern versuchen, das Faszinosum gewesen, das auch in die westliche Welt abgestrahlt hat (Stichwort OccupyBewegung). Dass die sozialen Bewegungen in den arabischen Ländern in ähnlicher Weise kollabieren, wie dies etwa mit dem Neuen Forum in der DDR nach der Wiedervereinigung geschah, ist hingegen recht unwahrscheinlich. In den tunesischen und ägyptischen sozialen Bewegungen ist nach den spontanen Aufständen des letzten Jahres gegenwärtig eine erhebliche Professionalisierung erkennbar. Aktivisten sind heute Kunden von Werbeagenturen, politische Kleinstgemeinschaften fordern basisdemokratische Rechte ein. Demonstrationen und Forderungen an die Übergangsregierungen Tunesiens und Ägyptens, die Demokratisierung voranzutreiben, sind demnach auch weniger Alarmsignale eines demokratischen Scheiterns als vielmehr positive Anzeichen der Wachsamkeit arabischer Gesellschaften. Diese wollen die einmalige historische Chance, die sich ihnen bietet, nicht verstreichen lassen. Dabei steht die Transition zur Demokratie leider erneut vor einem zentralen

12 Hafez, Kai (2011), Plädoyer für eine neue (Nah-)Ostpolitik, in: Neue Gesellschaft/Frankfurter Hefte 5, S. 13-18.
Dilemma: Einerseits ist die Bildung von politischen Parteien wichtig, um die Institutionen der Demokratie zu formen, andererseits ist die Fortexistenz sozialer Bewegungen nötig. Beide Prozesse behindern sich teilweise, weil die Energien gerade im Bereich der säkularen Parteigründungen noch fehlen oder nicht hinreichend gebündelt werden.

Für die Entwicklung der Demokratie in der arabischen Welt wird es nicht nur wichtig sein, dass die sozialen und politischen Bewegungen aktiv bleiben und sich Medien und politische Parteien weiter entwickeln, sondern dass sich auch das Band zwischen der demokratieorientierten Mehrheit der arabischen Bevölkerung und den neu entstandenen sozialen Bewegungen stabilisiert. An dieser Stelle muss man darauf hinweisen, dass die politische Transformation für viele arabische Bürger nicht nur Fortschritt und Hoffnung bedeutet, sondern auch mit erheblichen persönlichen Opfern verbunden ist, da sich die Lebensbedingungen im Alltag oft zunächst einmal verschlechtert haben. Bei Transformationen ist es häufig der Fall, dass die alten politischen Systeme nicht mehr greifen, die neuen aber noch nicht in Stellung gebracht sind, was wirtschaftliche Notsituationen hervorbringen kann, aber auch gesteigerte Sicherheitsprobleme und eine wachsende Verbrechensrate. Bei aller Bewunderung für die Jugendbewegungen des Internets und die Aktivisten der sozialen Bewegungen, die seit einem Jahr etwa an zentralen Orten wie dem Tahrir-Platz in Kairo ausharren, muss noch einmal betont werden, dass ohne die Mobilisierung weiterer Bevölkerungsmassen in den entscheidenden Tagen des Regimeumsturzes dieser nicht hätte gelingen können. Aus aktuellen Umfragen und unterschiedlichen Meinungsbildern in Tunesien und Ägypten aber kann man entnehmen, dass viele Bürger nicht nur von ihren Interimsregierungen, sondern auch von der Demonstrationslust der sozialen Bewegungen "genervt" sind. ${ }^{13}$ Die meisten Menschen wollen keine Basisdemokratie, sondern ordentliche repräsentative Demokratien. Sie verlangen nach einem neuen Staat, aber auch mit alten moralischen Sicherheiten. Es wäre fatal für die arabischen Demokratisierungsprozesse, wenn sich die breite Bevölkerungsmehrheit und die politische Kultur als Ganzes von der Revolution und von den sozialen Bewegungen abwenden würden. Der entscheidende Druck auf den autoritären Staat konnte nur durch das enge Bündnis zwischen den unterschiedlichen politischen und medialen Akteuren und den Wertekonsens in den Bevölkerungen erreicht werden. Auch die Protestierenden müssen daher mit Fingerspitzengefühl vorgehen - vielleicht wäre gelegentlich etwas weniger außerparlamentarische Aktivität und etwas mehr Parteiarbeit hilfreich. Ein Keil zwischen den sozialen Bewegungen und den Bevölkerungsmehrheiten wäre das Schlimmste, was passieren könnte. Dazu allerdings wird es aller Wahrscheinlichkeit nach letztlich nicht kommen.

13 al-Gawhary, Karim, Im Zweifel für das Bewährte, die tageszeitung, 21. November 2011. 